\title{
Learning Multi-level Region Consistency with Dense Multi-label Networks for Semantic Segmentation
}

\author{
Tong Shen ${ }^{1}$, Guosheng Lin $^{2 *}$, Chunhua Shen ${ }^{1}$, Ian Reid ${ }^{1}$ \\ ${ }^{1}$ School of Computer Science, The University of Adelaide, Australia \\ ${ }^{2}$ School of Computer Science and Engineering, Nanyang Technological University, Singapore \\ $\{$ tong.shen, chunhua.shen, ian.reid $\} @$ adelaide.edu.au \\ guosheng.lin@gmail.com
}

\begin{abstract}
Semantic image segmentation is a fundamental task in image understanding. Per-pixel semantic labelling of an image benefits greatly from the ability to consider region consistency both locally and globally. However, many Fully Convolutional Network based methods do not impose such consistency, which may give rise to noisy and implausible predictions. We address this issue by proposing a dense multi-label network module that is able to encourage the region consistency at different levels. This simple but effective module can be easily integrated into any semantic segmentation systems. With comprehensive experiments, we show that the dense multi-label can successfully remove the implausible labels and clear the confusion so as to boost the performance of semantic segmentation systems.
\end{abstract}

\section{Introduction}

Semantic segmentation is one of the fundamental problems in computer vision, whose task is to assign a semantic label to each pixel of an image so that different classes can be distinguished. This topic has been widely studied [Girshick $e t$ al., 2014; Hariharan et al., 2014; Yadollahpour et al., 2013; Farabet et al., 2013]. Among these models, Fully Convolutional Network (FCN) based models have become dominant [Dai et al., 2015; Chen et al., 2015; Lin et al., 2015; Chen et al., 2016]. These models are simple and effective because of the powerful capacity of Convolutional Neural Networks (CNNs) and being able to be trained end-to-end. However, most existing methods do not have the mechanism to enforce the region consistency, which plays an important role in semantic segmentation. Consider, for example, Figure 1, in which the lower left image is the output of a vanilla FCN, whose prediction contains some noisy labels that do not appear in the ground truth. With enforced region consistency, we can simply eliminate those implausible labels and clear the confusion. Our aim in this work is to introduce constraints to encourage this consistency.

${ }^{*}$ The first two authors contributed equally and this work was done when Guosheng Lin was with The University of Adelaide.

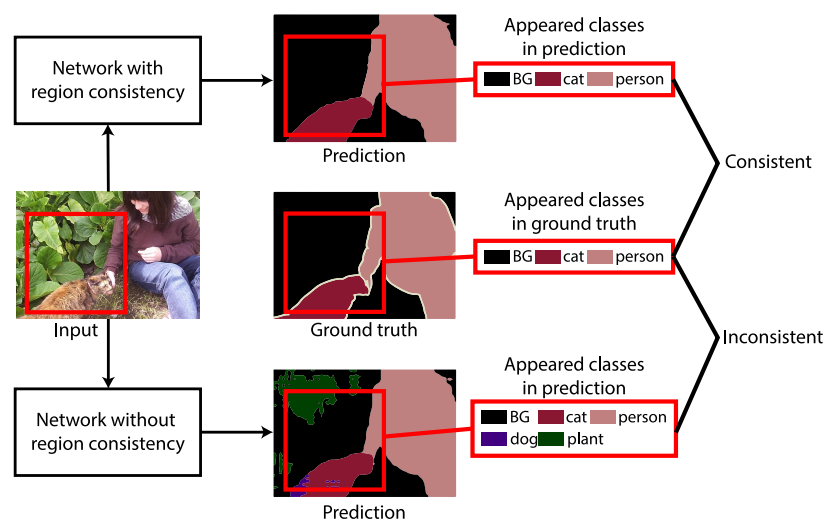

Figure 1: Illustration of region consistency. For a region in the input image, which is coloured in red, the corresponding part in the ground truth contains only three classes. In the network without region consistency, there are five classes that appear. If we explicitly encourage the consistency, those unlikely classes will be eliminated and the prediction will be better as shown on top.

Our proposal is both simple and effective: we argue that the region consistency in a certain region can be formulated as a multi-label classification problem. Multi-label classification has also been widely studied [Jiang, 2016; Wei et al., 2016; Guo and Gu, 2011], whose task is to assign one or more labels to the image. By performing multi-label classification in a region, we can allow the data to suggest which labels are likely within the broad context of the region, and use this information to suppress implausible classes predicted without reference to the broader context, thereby improving scene consistency. While typical multi-label problems are formulated as whole-image inference, we adapt this approach to dense prediction problems such as semantic segmentation, by introducing dense multi-label prediction for image regions of various sizes.

Dense multi-label prediction is performed in a sliding window fashion: the classification for each spatial point is influenced by the network prediction and by the multi-label result for the surrounding window. By employing different window sizes, we are able to construct a multi-level structure for dense multi-label and enforce the region consistency at different levels both locally and globally. Figure 2 is an illus- 

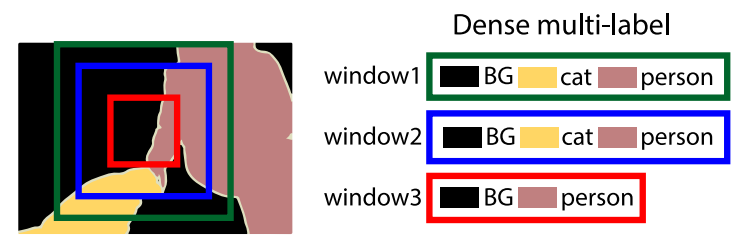

Figure 2: Illustration of dense multi-label with multi-level. Windows in different colours indicate different regions for dense multilabel classification.

tration of dense multi-label at multiple windows sizes.Here we use three windows of different sizes. The red window, the smallest, focuses more on the local region consistency, while the green window, the largest, is responsible for global region consistency. The other one, in blue, is for mid-level consistency. By sliding the windows to consider each spatial point, we perform multi-label densely at different level, encouraging the segmentation predictor to give predictions that are consistent with the dense multi-label prediction.

Our contributions are as follows:

- We address the problem of region consistency in semantic segmentation by proposing a dense multi-label module to achieve the goal of retaining region consistency, which is simple and effective. We also introduce a multilevel structure for dense multi-label to preserve region consistency both locally and globally.

- We evaluate our method on four popular semantic segmentation datasets including NYUDv2, SUN-RGBD, PASCAL-Context and ADE 20k, and achieve promising results. We also give analysis on how dense multi-label can remove the implausible labels, clear confusion and effectively boost the segmentation systems.

\section{Related Work}

Semantic segmentation has been widely studied [Girshick $e t$ al., 2014; Hariharan et al., 2014]. Early CNN based methods rely on region proposals or superpixels. They make segmentation prediction by classifying these local features. More recently, with Long et al.[Long et al., 2015] introducing applying Fully Convolutional Networks(FCNs) to semantic segmentation, the FCN based segmentation models [Dai et al., 2015; Chen et al., 2015; Lin et al., 2015; Chen et al., 2016] have become popular.

Multi-label classification has also been widely studied. Traditional methods are based on graphical models [Xue et al., 2011; Guo and Gu, 2011], while the recent studies benefit more from CNNs [Wei et al., 2016; Jiang, 2016].

Here we propose a dense multi-label module to take advantage of multi-label classification and integrate it into semantic segmentation systems. Dense multi-label is performed in a sliding window fashion and treats all area in a window as multi-label classification. Experiments show that dense multi-label can help to keep the scene consistency, clear confusion and boost the performance of semantic segmentation.

\section{Methods}

\subsection{Dense Multi-label}
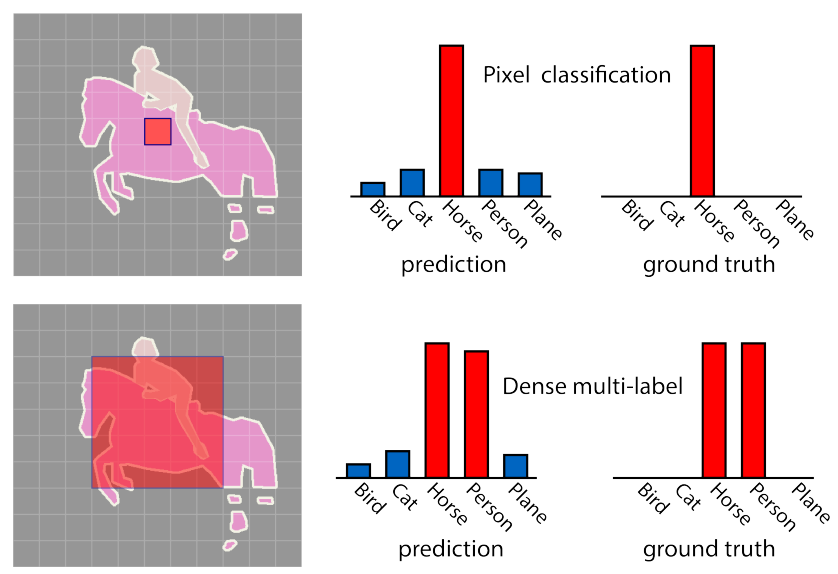

Figure 3: An illustration of differences between pixel classification and dense multi-label prediction. In pixel classification, we treat each spatial point as a single-label classification problem where only one class is supposed to get very high confidence; dense multi-label focuses on label concurrence where the labels that appear in the region will have equally high confidence.

Multi-label classification is a task where each image can have more than one label, unlike a multi-class classification problem [Simonyan and Zisserman, 2015; Szegedy et al., ; He et al., 2016] whose goal is to assign only one label to the image. This is more natural in reality because for majority of images, objects are not isolated, instead they are in context with other objects or the scene. Multi-label classification gives us more information of the image.

For a dense prediction task such as segmentation, it treats every spatial point as a multi-class classification problem, where the point is assigned with one of the categories. As shown in the upper part of Figure 3, the model predicts scores for each class and picks the highest one. The ground truth is an one-hot vector correspondingly. For a dense multi-label problem, each spatial point will be assigned with several labels to show what labels appear in the a certain window centered at this point. As shown in lower part of Figure 3, there are two classes being predicted with high confidence and the ground truth is given by a "multiple hot" vector.

Here we propose a method to learn a dense multi-label system and a segmentation system at the same time. We aim at using dense multi-label to suppress the implausible classes and encourage appropriate classes so as to retain the region consistency for the segmentation prediction both globally and locally. In the next section, more details of the whole framework will be provided.

\subsection{Overview of Framework}

An overview of the structure is shown in Figure 4, with the part in the dashed-line rectangle being the dense multi-label module. Without it, the network simply becomes a FCN. The input image is first fed into several low level feature layers 


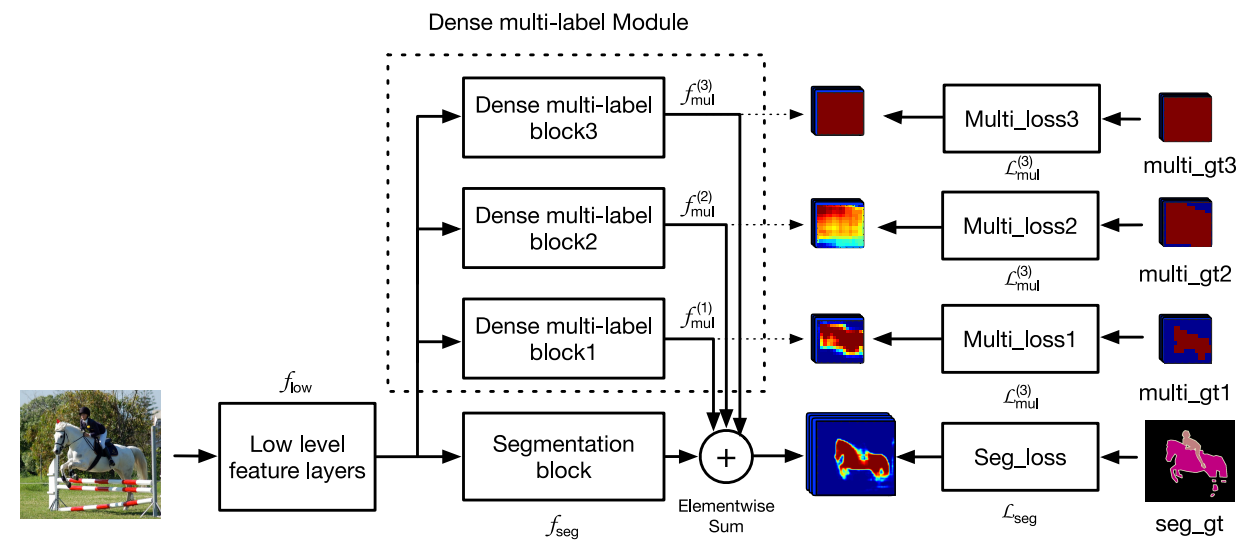

Figure 4: Illustration of the framework with dense multi-label module. The input image is first passed into low level feature layers, which are shared by the following blocks. Then the feature maps are fed into the segmentation block and three dense multi-label blocks. The element-wise sum will sum up the features from the blocks and make the final prediction. Apart from the segmentation loss, each dense multi-label block also has its own multi-label loss to guide the training.

that are shared by the following blocks. Then apart from going into the segmentation block, the features also enter three blocks for dense multi-label prediction. The outputs of theses blocks are merged element-wise for the final prediction.

In the training phase, the network is guided by four loss functions: the segmentation loss and three dense multi-label losses. We use softmax loss for the segmentation path, and use logistic loss for all the dense multi-label blocks.

The dense multi-label blocks have different window sizes for performing dense multi-label prediction within different contexts. With this multi-level structure, we are able to retain region consistency both locally and globally.

Let $\boldsymbol{x}$ denote the image. The process of the low level feature block can be described as:

$$
\boldsymbol{o}=f_{\text {low }}\left(\boldsymbol{x} ; \boldsymbol{\theta}_{\text {low }}\right),
$$

where $\boldsymbol{o}$ is the output and $\boldsymbol{\theta}_{\text {low }}$ the layer parameters.

The dense multi-label blocks and the segmentation block are defined as:

$$
\begin{gathered}
\boldsymbol{m}^{(j)}=f_{m u l}^{(j)}\left(\boldsymbol{o} ; \boldsymbol{\theta}_{m u l}^{(j)}\right), j \in\{1,2,3\} \\
\boldsymbol{s}=f_{\text {seg }}\left(\boldsymbol{o} ; \boldsymbol{\theta}_{\text {seg }}\right),
\end{gathered}
$$

where $\boldsymbol{m}^{(j)}$ and $s$ denote the output of $j$ th multi-label block and the output of segmentation respectively. $\boldsymbol{\theta}_{m u l}^{(j)}$ and $\boldsymbol{\theta}_{\text {seg }}$ are layer parameters.

The final prediction is:

$$
\boldsymbol{p}=\boldsymbol{s}+\boldsymbol{m}^{(1)}+\boldsymbol{m}^{(2)}+\boldsymbol{m}^{(3)},
$$

where $\boldsymbol{p}$ is the fused score for segmentation.

For the loss functions, we use logistic loss for the prediction of dense multi label blocks, $\boldsymbol{m}^{(1)}, \boldsymbol{m}^{(2)}$ and $\boldsymbol{m}^{(3)}$; softmax loss is used for final prediction $\boldsymbol{p}$. Let $m_{i k}$ be the out of a dense multi-label block at $i$ th position for $k$ th class, and $y_{i k}^{m u l}$ be the ground truth for the corresponding position and class. The loss function for dense multi-label is defined as:

$$
\begin{aligned}
l_{m u l}\left(\boldsymbol{y}^{m u l}, \boldsymbol{m}\right) & =\frac{1}{I K} \sum_{i}^{I} \sum_{k}^{K} y_{i k}^{m u l} \log \left(\frac{1}{1+e^{-m_{i k}}}\right) \\
& +\left(1-y_{i k}^{m u l}\right) \log \left(\frac{e^{-m_{i k}}}{1+e^{-m_{i k}}}\right),
\end{aligned}
$$

where $y_{i k}^{m u l} \in\{0,1\} ; I$ and $K$ represent the number of spatial points and classes, respectively.

Similarly, let $p_{i k}$ be the fused output at $i$ th position for $k$ th class, and $y_{i}^{s e g}$ be the ground truth for segmentation prediction at $i$ th position. The loss function for segmentation is defined as:

$$
l_{\text {seg }}\left(\boldsymbol{y}^{\text {seg }}, \boldsymbol{p}\right)=\frac{1}{I} \sum_{i}^{I} \sum_{k}^{K} \mathbb{I}\left(y_{i}^{\text {seg }}=k\right) \log \left(\frac{e^{p_{i k}}}{\sum_{j} e^{p_{i j}}}\right),
$$

where $y_{i}^{\text {seg }} \in\{1 \ldots K\}$.

Our goal is to minimize the objective function:

$$
\min l_{\text {seg }}+\lambda\left(l_{\text {mul }}^{(1)}+l_{m u l}^{(2)}+l_{m u l}^{(3)}\right),
$$

where $\lambda$ controls the balance between the segmentation block and the dense multi-label blocks. I observe this parameter is not very sensitive. We set $\lambda=1$ to treat each part equally.

\subsection{Dense Multi-label Block}

The details of the dense multi-label block are shown in Figure 5 , where the input is feature maps at $1 / 8$ resolution, due to the downsampling in the low level feature layers. After some convolutional layers with further downsampling, the dense multi-label is performed at $1 / 32$ resolution with the sliding window and following adaptive layers. The reason for this setting is because dense multi-label requires a large sliding window, which will become a computational burden if we work at a high resolution. Downsampling can greatly reduce the size of feature maps and more importantly, the size of sliding window will shrink accordingly, thus making the computation more efficient. On the other hand, dense multi-label 
requires more high level information. Therefore, working at a coarse level can capture the high level features better. The output of the dense multi-label is upsampled to be compatible with the segmentation block's output.

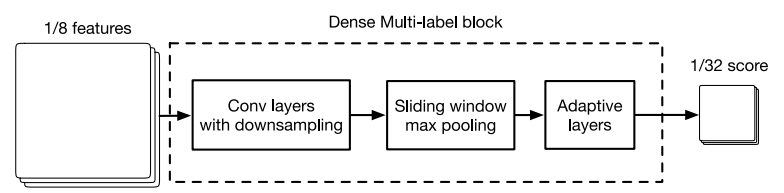

Figure 5: Details of a single dense multi-label block. The input features are fed into several convolutional layers and further downsampled. Then we perform sliding window with max pooling operation. After some adaptive layers, we have scores for dense multi-label at $1 / 32$ resolution.

\subsection{Ground Truth Generation}

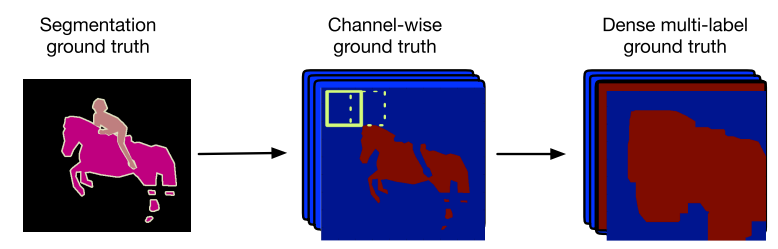

Figure 6: The segmentation ground truth is firstly converted to channel-wise labels, with 0 or 1 in each channel. The ground truth for dense multi-label can be obtained by performing max pooling on the channel-wise labels.

The ground truth for dense multi-label can be generated from the segmentation ground truth. The process is described in Figure 6. Firstly, the segmentation ground truth is converted to channel-wise labels, which means each channel only contains 1 or 0 to indicate whether the corresponding class appears or not. To generate a ground-truth mask for each class, for a given window size, we slide the window across each binary channel and perform a max-pool operation (this is equivalent to a binary dilation using a structuring element of the same size and shape as the window). We repeat this process for each window size. As noted in section 3.3, the dense multi-label classification is performed at 1/32 resolution while the segmentation is at $1 / 8$. Therefore, we generate multi-label ground-truth data at $1 / 8$ resolution with stride 4 .

\subsection{Network Configuration}

The dense multi-label module is suitable for any segmentation system and it can be easily integrated. In this study, we use Residual 50-layer network [He et al., 2016] with dilated kernels [Chen et al., 2015]. In order to work at a relatively high resolution while keeping the efficiency, we use 8-stride setting, which means that the final output is at $1 / 8$ resolution. As we mentioned in the last section, we perform dense multilabel at 1/32 resolution to make it more efficient and effective. The window sizes are then defined at $1 / 32$ resolution. For example,let $w$ be the window size. A window with $w=17$ at

\begin{tabular}{|c|c|c|}
\hline Block name & Initial layers & Stride \\
\hline \hline Low level feature block & conv1 to res3d & 8 \\
\hline Segmentation block & res4a to res5c & 1 \\
\hline Dense multi-label block & res4a to res5c & 4 \\
\hline
\end{tabular}

Table 1: Configuration for Res50 network. The low level feature block is initialized by layers "conv1" to "res3d" and has 8 stride. The segmentation block and dense multi-label blocks are initialized by layers "res4a" to "res5c" but do not share the weights with each other. The segmentation block does not have any downsampling, but the dense multi-label blocks have further 4 stride downsampling.

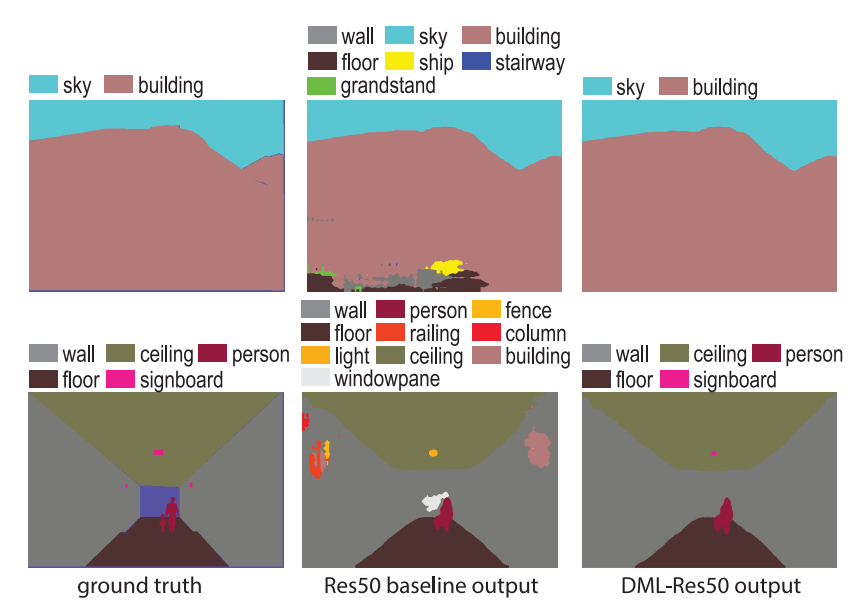

Figure 7: Example outputs of Res50 baseline and DML-Res50 on ADE-20k dataset.

$1 / 32$ resolution means $4 w=68$ at $1 / 8$ resolution. The corresponding window for the original image is $32 w=544$. We use $w_{1}=35, w_{2}=17$ and $w_{3}=7$ for all the experiments.

Table 1 shows the configuration with 50-layer Residual net (Res50) as the base network. The low level feature block contains layers from "conv1" to "res3d". The segmentation block and dense multi-label blocks have layers from "res $4 a$ " to "res5c" as well as some adaptive layers. It is worth noting that it does not mean these blocks will share the weights even though they initialize the weights from the same layers. After initialization, they will learn their own features separately.

\section{Experiments and Analysis}

We evaluate our model on 4 commonly used semantic segmentation datasets: ADE-20k, NYUDv2, SUN-RGBD and PASCAL-Context. Our comprehensive experiments show that dense multi-label can successfully suppress many unlikely labels, retain region consistency and thus improve the performance of semantic segmentation.

The results are evaluated using the Intersection-over-Union (IoU) score [Everingham et al., 2010]. Moreover, since our original motivation is to suppress noisy and unreasonable labels to keep labels consistent with the region, we also introduce new measurements to evaluate the number of classes that are not in ground truth, and further, the number of pixels that are predicted to be these wrong classes for each image.

We only use Res50 as base network to compare and analyse the performance. For all the experiments, we use batch size 


\begin{tabular}{|c|c|c|c|}
\hline Model & IOU & \#Wrong class & \#Wrong label \\
\hline \hline Res50 baseline & 34.5 & 5.6 & 21836 \\
\hline DML Res50 & $\mathbf{3 6 . 5}$ & $\mathbf{3 . 6}$ & $\mathbf{1 8 2 9 4}$ \\
\hline
\end{tabular}

Table 2: Results on ADE-20k. The dense multi-label boosts the IOU by $2 \%$ and helps reduce the number of wrong class and label by $35 \%$ and $16 \%$ respectively.

\begin{tabular}{|c|c|}
\hline Model & IOU \\
\hline \hline DilatedNet [Zhou et al., 2016] & 32.3 \\
\hline Cascade-DilatedNet [Zhou et al., 2016] & 34.9 \\
\hline DML-Res50(ours) & $\mathbf{3 6 . 5}$ \\
\hline
\end{tabular}

Table 3: Comparsion with other models on ADE-20k dataset. Our model achieves the best performance.

of 8 , momentum of 0.9 and weight decay of 0.0005 .

\subsection{Results on ADE-20k}

We first evaluate our result on ADE-20k dataset[Zhou et al., 2016], which contains 150 semantic categories including objects such as person, car etc., and "stuff" such as sky, road etc. . There are 20210 images in the training set and 2000 images in the validation set.

As shown in Table 2, the model with dense multi-label (DML-Res50) yields a $2 \%$ improvement. To analyse the effectiveness of label suppression, we also use two criteria to evaluate this performance, which are shown as "Wrong class" and "Wrong labels". Wrong class means the number classes that are not supposed to appear but are mistakenly predicted by the model. Wrong labels describe how many pixels are assigned with those wrong classes. We observe that using Dense multi-label effectively reduces the wrong classes and labels, by $35 \%$ and $16 \%$ respectively. Some examples are shown in Figure 7. To make fair comparison, all the images are raw outputs directly from the network. The last column shows the outputs from the network with dense multi-label where we can observe great scene consistency compared with the output of the baseline network shown in the middle.

We achieve better results than the models reported in [Zhou et al., 2016], as shown in Table 3.

\subsection{Results on PASCAL-Context}

PASCAL-Context dataset [Mottaghi et al., 2010] is a set of additional annotations for PASCAL VOC 2010, which provides annotations for the whole scene with 60 classes (59 classes and a background class). It contains 4998 images in training set and 5105 images in validation set.

Figure 8 shows some typical examples on this dataset. We can also see clear scene consistency with dense multi-label involved. The outputs in the middle contain many noisy classes, especially the lower middle image contains "bird" and "sky", which are very unlikely in this scene. From Table 4, we can also see the great boost with dense multi-label. The wrong classes and labels are greatly reduced by $37 \%$ and $15 \%$.

To compare with other models, we list several results on this dataset. Since different models have various settings such as multi-scale training, extra data, etc.we also explain it in Table 5. Considering all the factors involved, our method is

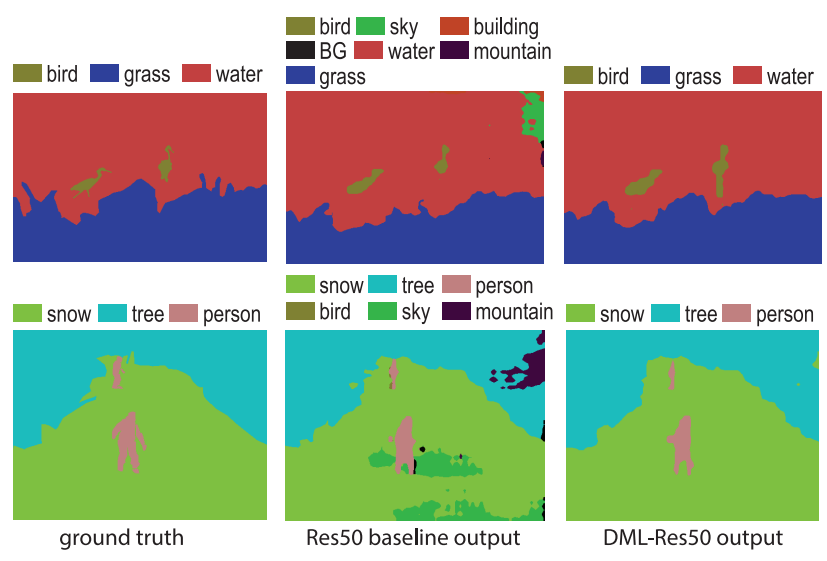

Figure 8: Example outputs of Res50 baseline and DML-Res50 on PASCAL-Context dataset.

\begin{tabular}{|c|c|c|c|}
\hline Model & IOU & \#Wrong class & \#Wrong label \\
\hline \hline Res50 baseline & 41.4 & 4.5 & 26308 \\
\hline DML-Res50 & $\mathbf{4 4 . 4}$ & $\mathbf{2 . 8}$ & $\mathbf{2 2 3 6 7}$ \\
\hline
\end{tabular}

Table 4: Results on PASCAL-Context dataset. The dense multilabel model increases the IOU by $3 \%$ and reduces the wrong classes and labels by $37 \%$ and $15 \%$.

comparable since we only use Res50 as the base network and do not use multi-scale training and extra MS-COCO data.

\subsection{Results on NYUDv2}

NYUDv2 [Silberman et al., 2012] is comprised of $1449 \mathrm{im}$ ages from a variety of indoor scenes. We use the standard split of 795 training images and 654 testing images.

Table 6 shows the results on this dataset. With dense multilabel, the performance is improved by more than $1 \%$, and the number of wrong class and label decrease by about $40 \%$ and $16 \%$. Some examples are shown in Figure 9. Scene consistency still plays an important role in removing those noisy labels. Compared with some other models, we achieve the best result, as shown in Table 7.

\subsection{Results on SUN-RGBD}

SUN-RGBD [Song et al., 2015] is an extension of NYUDv2 [Silberman et al., 2012], which contains 5285 training images and 5050 validation images, and provides pixel labelling masks for 37 classes.

Figure 10 shows some output comparison on this dataset, where we can easily observe the effect of dense multi-label. The results are shown in Table 8. The network with dense multi-label helps improve the IOU by more than $3 \%$. The wrong classes and labels also get decreased by $36 \%$ and $18 \%$ respectively. Compared with other methods, the network with dense multi-label reaches the best result, as shown in Table 9.

\subsection{Ablation Study on PASCAL-Context}

Table 10 shows an ablation study on the PASCAL-Context. The Res50 baseline yields mean IOU of $41.4 \%$. Treating this as a baseline, we introduce dense multi-level module. Firstly, in the one level setting, we use the largest window 


\begin{tabular}{|c|c|c|c|c|}
\hline Model & Base & MS & Ex data & IOU \\
\hline \hline FCN-8s [Long et al., 2015] & VGG16 & no & no & 37.8 \\
\hline PaserNet [Liu et al., 2015] & VGG16 & no & no & 40.4 \\
\hline HO_CRF [Arnab et al., 2015] & VGG16 & no & no & 41.3 \\
\hline Context [Lin et al., 2016] & VGG16 & yes & no & 43.3 \\
\hline VeryDeep [Wu et al., 2016] & Res101 & no & no & 44.5 \\
\hline DeepLab [Chen et al., 2016] & Res101 & yes & COCO & $\mathbf{4 5 . 7}$ \\
\hline DML-Res50 (ours) & Res50 & no & no & 44.4 \\
\hline
\end{tabular}

Table 5: Results on PASCAL-Context dataset. MS means using multi-scale inputs and fusing the results in training. Ex data stands for using extra data such as MS-COCO [Lin et al., 2014]. Compared with state of the art, since we only use Res50 instead of Res101 and do not use multi-scale training as well as extra data, our result is comparable.

\begin{tabular}{|c|c|c|c|}
\hline Model & IOU & \#Wrong class & \#Wrong label \\
\hline \hline Res50 baseline & 38.8 & 8.2 & 27577 \\
\hline DML-Res50 & $\mathbf{4 0 . 2}$ & $\mathbf{4 . 9}$ & $\mathbf{2 3 0 5 7}$ \\
\hline
\end{tabular}

Table 6: Results on NYUDv2 dataset. Dense multi-label network has $1.4 \%$ higher IOU and $40 \%$ and $16 \%$ lower wrong classes and labels respectively.

size, which is basically global multi-label classification. Accordding to the results, the first level gives the biggest boost. With 2 levels involved, the global and mid-level window, the performance is improved further. The final level, the smallest window, brings $0.6 \%$ more improvement. The dense multilabel module helps improve the performance by $2.2 \%$ in total. After using CRF as post-processing, we can achieve IOU of 44.4 without using extra MS COCO dataset.

\begin{tabular}{|c|c|}
\hline Model & IOU \\
\hline \hline FCN-32s [Long et al., 2015] & 29.2 \\
\hline FCN-HHA [Long et al., 2015] & 34.0 \\
\hline Context [Lin et al., 2016] & 40.0 \\
\hline DML-Res50 (ours) & 40.2 \\
\hline
\end{tabular}

Table 7: Comparison with other models on NYUDv2 dataset. Our method achieves the best result.

\section{Conclusion}

In this study, we propose a dense multi-label module to address the problem of scene consistency. With comprehensive experiments, we have shown that dense multi-label can enforce the scene consistency in a simple and effective way. More importantly, the dense multi-label is a module and can be easily integrated into other semantic segmentation systems.

\section{Acknowledgements}

This research was supported by the Australian Research Council through the Australian Centre for Robotic Vision (CE140100016). C. Shen's participation was supported by an ARC Future Fellowship (FT120100969). I. Reid's participation was supported by an ARC Laureate Fellowship (FL130100102).
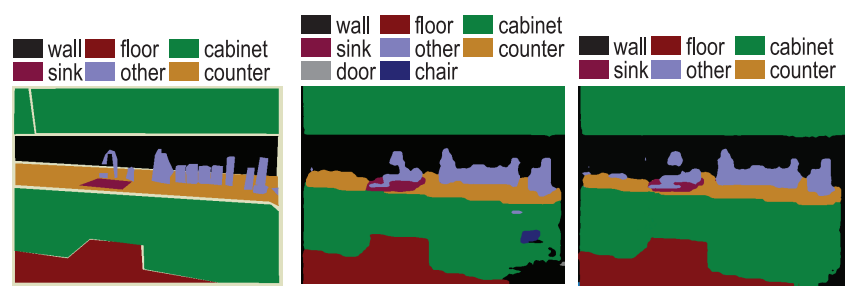

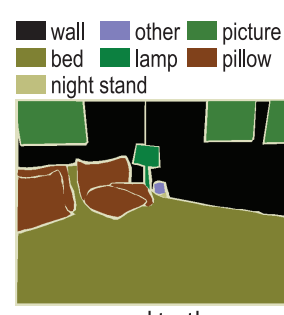

ground truth

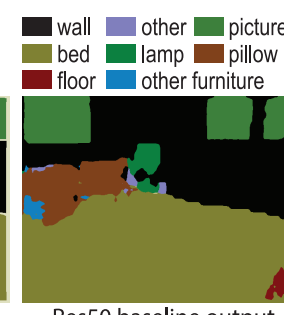

Res50 baseline output

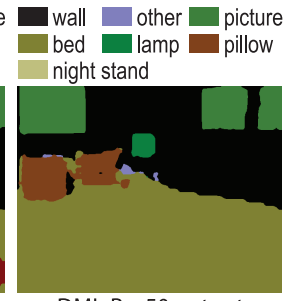

DML-Res50 output
Figure 9: Example outputs of Res50 baseline and DML-Res50 on NYUDv2 dataset.

\begin{tabular}{|c|c|c|c|}
\hline Model & IOU & \#Wrong class & \#Wrong label \\
\hline \hline Res50 baseline & 39.3 & 5.3 & 24602 \\
\hline DML-Res50 & $\mathbf{4 2 . 3}$ & $\mathbf{3 . 4}$ & $\mathbf{2 0 1 0 4}$ \\
\hline
\end{tabular}

Table 8: Results on SUN-RGBD dataset. Dense multi-label helps increase the performance by more than $3 \%$ of IOU and decrease the wrong classes and labels by $36 \%$ and $18 \%$.

\begin{tabular}{|c|c|}
\hline Model & IOU \\
\hline \hline Kendall et al.[Kendall et al., 2015] & 30.7 \\
\hline Context [Lin et al., 2016] & 42.3 \\
\hline DML-Res50 (ours) & 42.4 \\
\hline
\end{tabular}

Table 9: Comparison with other models on SUN-RGBD dataset. We achieve the best result with dense multi-label network.
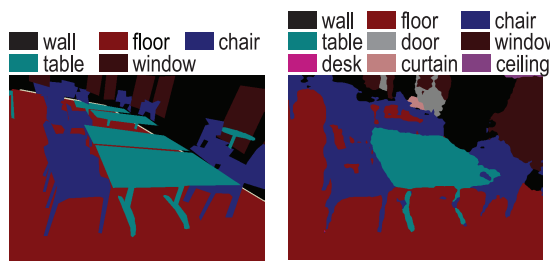

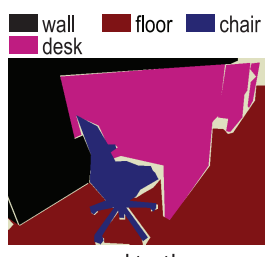

ground truth

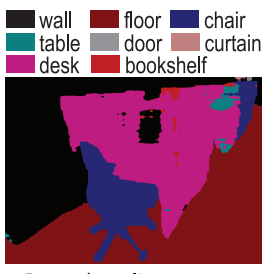

Res50 baseline output
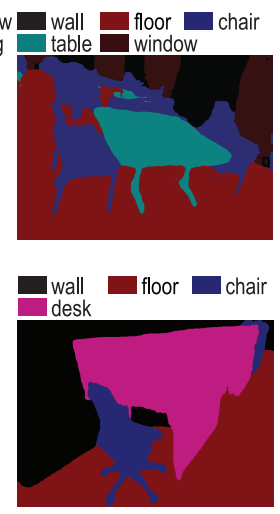

DML-Res50 output
Figure 10: Example outputs of Res50 baseline and DML-Res50 on SUN-RGBD dataset.

\begin{tabular}{|c|c|}
\hline Model & IOU \\
\hline \hline Res50 baseline & 41.4 \\
\hline DML-Res50 1level & 42.5 \\
\hline DML-Res50 2level & 43.0 \\
\hline DML-Res50 3level & 43.6 \\
\hline DML-Res50 3level + CRF & $\mathbf{4 4 . 4}$ \\
\hline
\end{tabular}

Table 10: Ablation study on PASCAL-Context. 


\section{References}

[Arnab et al., 2015] Anurag Arnab, Sadeep Jayasumana, Shuai Zheng, and Philip Torr. Higher Order Conditional Random Fields in Deep Neural Networks. Arxiv, page 10, 2015.

[Chen et al., 2015] Liang-Chieh Chen, George Papandreou, Iasonas Kokkinos, Kevin Murphy, and Alan L. Yuille. Semantic Image Segmentation with Deep Convolutional Nets and Fully Connected CRFs. In ICLR, 2015.

[Chen et al., 2016] Liang-Chieh Chen, George Papandreou, Iasonas Kokkinos, Kevin Murphy, and Alan L. Yuille. DeepLab: Semantic Image Segmentation with Deep Convolutional Nets, Atrous Convolution, and Fully Connected CRFs. CoRR, abs/1606.0, 2016.

[Dai et al., 2015] Jifeng Dai, Kaiming He, and Jian Sun. [M] BoxSup: Exploiting Bounding Boxes to Supervise Convolutional Networks for Semantic Segmentation. In ICCV, pages 1635-1643, 2015.

[Everingham et al., 2010] Mark Everingham, Luc Van Gool, Christopher K I Williams, John Winn, and Andrew Zisserman. The pascal visual object classes (VOC) challenge. International Journal of Computer Vision, 88(2):303-338, 2010.

[Farabet et al., 2013] Clément Farabet, Camille Couprie, Laurent Najman, and Yann Lecun. Learning Hierarchical Features for Scene Labeling. TPAMI, 35(8):1915-1929, 2013.

[Girshick et al., 2014] Ross Girshick, Jeff Donahue, Trevor Darrell, and Jitendra Malik. Rich Feature Hierarchies for Accurate Object Detection and Semantic Segmentation. In CVPR, pages 580-587, 2014.

[Guo and Gu, 2011] Yuhong Guo and Suicheng Gu. Multilabel classification using conditional dependency networks. In IJCAI, pages 1300-1305, 2011.

[Hariharan et al., 2014] Bharath Hariharan, Pablo Arbeláez, Ross Girshick, and Jitendra Malik. Simultaneous Detection and Segmentation. ECCV, pages 297-312, 2014.

[He et al., 2016] Kaiming He, Xiangyu Zhang, SHaoqing Ren, and Jian Sun. Deep Residual Learning for Image Recognition. In CVPR, pages 770-778, 2016.

[Jiang, 2016] Wang Jiang. CNN-RNN : A Unified Framework for Multi-label Image Classification. CVPR, 2016.

[Kendall et al., 2015] Alex Kendall, Vijay Badrinarayanan, and Roberto Cipolla. Bayesian SegNet: model uncertainty in deep convolutional encoder-decoder architectures for scene understanding. arXiv:1511.02680v1 [cs.CV], 2015.

[Lin et al., 2014] Tsung-Yi Lin, Michael Maire, Serge Belongie, Lubomir D Bourdev, Ross B Girshick, James Hays, Pietro Perona, Deva Ramanan, Piotr Dollár, and C Lawrence Zitnick. Microsoft \{COCO:\} Common Objects in Context. \{arXiv\}:1405.0312, pages 740-755, 2014.
[Lin et al., 2015] Guosheng Lin, Chunhua Shen, Anton van dan Hengel, and Ian Reid. Efficient piecewise training of deep structured models for semantic segmentation. pages 1-13, apr 2015.

[Lin et al., 2016] Guosheng Lin, Chunhua Shen, Anton Van Den Hengel, and Ian Reid. Exploring Context with Deep Structured models for Semantic Segmentation. Arxiv 2016, pages 1-14, 2016.

[Liu et al., 2015] Wei Liu, Andrew Rabinovich, and Alexander C. Berg. ParseNet: Looking Wider to See Better. arXiv preprint: arXiv:1506.04579, pages 1-11, 2015.

[Long et al., 2015] Jonathan Long, Evan Shelhamer, and Trevor Darrell. Fully Convolutional Networks for Semantic Segmentation. CVPR, pages 3431-3440, 2015.

[Mottaghi et al., 2010] Roozbeh Mottaghi, Xianjie Chen, Xiaobai Liu, Nam-gyu Cho, Seong-whan Lee, Raquel Urtasun, and Alan Yuille. The Role of Context for Object Detection and Semantic Segmentation in the Wild. 2010.

[Silberman et al., 2012] Nathan Silberman, Derek Hoiem, Pushmeet Kohli, and Rob Fergus. Indoor segmentation and support inference from RGBD images. Lecture Notes in Computer Science (including subseries Lecture Notes in Artificial Intelligence and Lecture Notes in Bioinformatics), 7576 LNCS(PART 5):746-760, 2012.

[Simonyan and Zisserman, 2015] Karen Simonyan and Andrew Zisserman. Very Deep Convolutional Networks for Large-Scale Image Recognition. pages 1-14, 2015.

[Song et al., 2015] Shuran Song, Samuel P. Lichtenberg, and Jianxiong Xiao. SUN RGB-D: A RGB-D scene understanding benchmark suite. CVPR, pages 567-576, 2015.

[Szegedy et al., ] Christian Szegedy, Sergey Ioffe, and Vincent Vanhoucke. Inception-v4, Inception-ResNet and the Impact of Residual Connections on Learning.

[Wei et al., 2016] Yunchao Wei, Wei Xia, Min Lin, Junshi Huang, Bingbing Ni, Jian Dong, Shuicheng Yan, and Yao Zhao. HCP: A Flexible CNN Framework for Multi-Label Image Classification. TPAMI, 38(2):1901-1907, 2016.

[Wu et al., 2016] Zifeng Wu, Chunhua Shen, and Anton Van Den Hengel. Bridging Category-level and Instancelevel Semantic Image Segmentation. 2016.

[Xue et al., 2011] Xiangyang Xue, Wei Zhang, Jie Zhang, Bin $\mathrm{Wu}$, Jianping Fan, and Yao Lu. Correlative multi-label multi-instance image annotation. ICCV, pages 651-658, 2011.

[Yadollahpour et al., 2013] Payman Yadollahpour, Dhruv Batra, and Gregory Shakhnarovich. Discriminative reranking of diverse segmentations. CVPR, pages 19231930, 2013.

[Zhou et al., 2016] Bolei Zhou, Hang Zhao, Xavier Puig, Sanja Fidler, Adela Barriuso, and Antonio Torralba. Semantic Understanding of Scenes through the ADE20K Dataset. arXiv, 2016. 